\title{
A PRECARIEDADE DOS REGISTROS DE ASSISTÊNCIA PRÉ-NATAL EM UMA UNIDADE BÁSICA DE SAÚDE NO MUNICÍPIO DE RIBEIRÃO PRETO - SP*
}

\author{
Maria das Dores do Vale Oba ** \\ Maria Solange Guarino Tavares***
}

OBA, M.das D.do V.; TAVARES, M.S.G. A precariedade dos registros de assistência pré-natal em uma unidade básica de saúde no município de Ribeirão Preto - SP. Rev.latino-am.enfermagem, Ribeirão Preto, v. 6, n. 1, p. 53-61, janeiro 1988.

Esta pesquisa deve como objetivo caracterizar a população de mulheres gestantes que procuram o serviço de atendimento pré-natal de uma Unidade Básica de Saúde do município de Ribeirão Preto - S.P. Brasil no mês de julho de 1995 e analisar o registro da assistência prestada às gestantes usuárias dessa Unidade, quanto a anamnese geral e específica, exame físico geral e tocoginecológico e procedimentos diagnósticos utilizados nesse serviço. Observa-se que os prontuários analisados deste serviço de atenção pré-natal apresentavam um registro em que inexiste a informação referente a anamnese geral e especifica, exame fisico geral e tocoginecológico e às condição clínica das gestantes, caracterizando um atendimento mais ritualístico. Desta forma, os registros não atendiam aos objetivos propostos pelo Programa de Assistência Integral a Saúde da Mulher da Secretaria Municipal da Saúde de Ribeirão Preto ${ }^{9}$. Secretaria de Estado da Saúde de São Paulo ${ }^{10,11}$ e do Ministério da Saúde ${ }^{3}$, a saber: assegurar a boa qualidade do atendimento à saúde da mulher quer nas suas necessidades clínicas, ginecológicas, obstétricas e mentais; identificar, prevenir e controlar os fatores de risco que possam afetar a saúde da mulher; diagnosticar e tratar o mais precocemente possivel as patologias da mulher, entre outros.

UNITERMOS: assistência, saúde da mulher

\section{INTRODUÇÃO}

Segundo DUGAS 5 , o registro representa a comunicação escrita dos fatores essenciais, de forma a manter uma história contínua dos acontecimentos ocorridos durante um certo período. Assim, o registro é um instrumento de comunicação importante na interligação entre os serviços, ou seja, é a constituição de uma memória. As informações registradas podem expressar o perfil epidemiológico do atendimento, a incidência e prevalência de determinados tipos de agravos na saúde, sendo material de referência e aperfeiçoamento para o ensino e pesquisa.

A experiência de analisar as informações existentes nos prontuários de um Unidade Básica de Saúde do município de Ribeirão Preto, que desenvolve o atendimento pré-natal, chama a nossa atenção a respeito da importância na elaboração de registros, em especial para este trabalho.
Os registros analisados encontram-se centrado na "incompreensibilidade", na "incompletude", ou seja, na falta das informações completas ou corretas na quase totalidade dos registros obtidos nos prontuários de assistência ao pré-natal. Neste caso questiona-se a qualidade destas informações para memória deste acervo.

Assim, objetivou-se:

- caracterizar a população de mulheres gestantes que procuram o serviço de atendimento pré-natal de uma Unidade Básica de Saúde do município de Ribeirão Preto, no mês de julho de 1995;

- analisar o registro da assistência prestada às gestantes usuárias dessa Unidade, quanto a anamnese geral e específica, exame físico geral e tocoginecológico e procedimentos diagnósticos utilizados nesse serviço.

Segundo TESTA et al. ${ }^{13}$, as práticas institucionais devem ser examinadas em todos os terrenos, onde a realidade adquire a consistência, através de sua organização, suas ações cotidianas e dos planos para o

\footnotetext{
* Dados do projeto piloto da dissertação de mestrado. Trabalho apresentado no $48^{\circ}$ Congresso Brasileiro de Enfermagem ** Enfermeira da SMS de Ribeirão Preto. Mestre em Enfermagem de Saúde Pública pela Escola de Enfermagem de Ribeirão Preto da Universidade de São Paulo e com especialização em Enfermagem do Trabalho, Saúde Pública e Administração Hospitalar *** Professora Doutora da área Saúde da Mulher do Departamento de Enfermagem Materno-Infantil e Saúde Pública da Escola de Enfermagem de Ribeirão Preto da Universidade de São Paulo
} 
futuro próximo e longínquo, entendidas como uma intermediação de classe e de direitos, próprio na definição das ações de saúde e das políticas sociais. Assim, os serviços de assistência à saúde devem ser analisados com o objetivo de obter informação da condição de estruturação da política de saúde vigente no país.

TANAKA $^{12}$, em seu estudo sobre a mortalidade materna, relata que alguns prontuários médicos ou fichas clínicas de serviço ambulatorial de atenção pré-natal apresentaram um registro precário ou mesmo inexistente, quanto às informações referentes às condições clínicas das gestantes.

A conferência internacional denominada "Safe Motherhood Conference" (maternidade sem risco) chamou atenção, em 1987, para a tragédia silenciosa das mortes maternas nos países subdesenvolvidos que, segundo LAURENTI ${ }^{6}$, ocorria no período em cerca de meio milhão de mulheres no mundo, sendo $90 \%$ delas nesses países. O mais terrível é que a maioria dessas mortes ou quase a totalidade delas eram preveníveis, pois a causa morte era decorrente de complicações obstétricas diretamente relacionadas à gestação, cujo atendimento de pré-natal poderia ajudar na sua redução, principalmente pelo acesso aos serviços de saúde e à boa qualidade da assistência.

Segundo o Manual dos Comitês de Mortalidade Materna do Ministério da Saúde ${ }^{2}$, os determinantes da mortalidade materna ou doenças do período gravídicopuerperal estão reunidos em dois grupos: as causas diretas, quais sejam, as toxemias gravídicas, o aborto, as alterações placentárias, alguns tipos de embolias pósparto e as hemorragias uterinas; as causas indiretas, que são aquelas doenças preexistentes, ou que surgem durante o período, e/ou agravadas por ele como cardiopatias, diabetes mellitus, nefropatias e doenças infecciosas. As causas obstétricas diretas podem ser prevenidas por uma boa assistência pré-natal, apesar de serem dependentes de outros determinantes, tais como a condição da mulher na sociedade e na família.

\section{METODOLOGIA}

Este estudo foi realizado em uma Unidade Básica de Saúde, localizada na região leste do município de Ribeirão Preto - S.P., tendo como amostra 53 prontuários de mulheres, que constituiu-se na totalidade de gestantes atendidas no mês de julho de 1995. O critério para inclusão destes no levantamento foi das gestantes terem sido atendidas neste serviço no período em estudo.
Utilizou-se um instrumento com a intenção de verificar a caracterização da clientela que utiliza o serviço de assistência pré-natal. O instrumento constou dos seguintes itens que foram levantados: as condições de admissão no pré-natal, os antecedentes obstétricos/ ginecológicos, dados referente a anamnese geral e específica, exame físico geral e tocoginecológico, imunização, procedimentos diagnósticos utilizados nesse serviço (exames laboratoriais, ultra-sonografia e citológico) e dados de identificação pessoal.

Considerando o propósito do trabalho, optou-se pela análise de conteúdo, segundo BARDIN ${ }^{1}$, uma vez que esta permite a representação e o tratamento dos dados de uma pesquisa, quer seja com abordagem qualitativa ou quantitativa.

O conteúdo analisado foi subdividido em categorias:

Caracterização da população - Identificação: Categoria I Identificação dos procedimentos realizados na assistência Pré-Natal: Categoria II.

\section{RESULTADOS}

Para melhor compreensão das informações contidas nos prontuários e do sistema de registro utilizado pela rede municipal de saúde de Ribeirão Preto, os dados foram analisados segundo a ordem da temática estudada.

\section{Caracterização da população - Identificação: Categoria I}

Nesta categoria procurou-se verificar a informação contida no prontuário de modo que fosse possível identificar a clientela de mulheres gestantes, que utilizaram o serviço de pré-natal de uma Unidade Básica de Saúde de Ribeirão Preto, no mês de julho de 1995 .

Observamos que em $100 \%$ da amostra não existia dados referentes à procedência, escolaridade, ocupação, relação de emprego, renda familiar, estado civil, tempo de residência e patologia anterior à gestação ou patologias de outras gestações.

Segundo DUGAS ${ }^{5}$, o registro deve incluir: nome completo, endereço, número de matrícula, data e hora de entrada, nome do médico, detalhes da responsabilidade financeira, sexo e estado civil, profissão e empregador, diagnóstico e atendimento anterior .

As Tabelas abaixo caracterizaram a clientela em estudo e o perfil de atendimento, conforme os dados registrados nos prontuários.

A amostra foi composta de 53 prontuários de gestantes na faixa etária de 14 a 34 anos, conforme Tabela 1. 
Tabela 1- Distribuição das gestantes segundo a idade, conforme dados dos prontuários*

\begin{tabular}{lcc}
\hline Idade & $\mathrm{n}$ & $\mathbf{F r}(\mathbf{\%})$ \\
\hline 14 a 20 anos & 8 & $\mathbf{1 5}$ \\
21 a 30 anos & 34 & $\mathbf{6 4}$ \\
31 a 34 anos & 11 & $\mathbf{2 1}$ \\
\hline TOTAL & 53 & $\mathbf{1 0 0}$ \\
$\begin{array}{l}\text { * Unidade Básica de Saúde no município de Ribeirão Preto, } \\
\text { julho 1995 }\end{array}$
\end{tabular}

Nesta pesquisa as gestantes deste serviço iniciaram os seus pré-natais no primeiro trimestre gestacional em 64\% (Tabela 2), período adequado para um levantamento das necessidades e/ou alterações que possam influenciar negativamente no transcorrer da própria gestação, do parto e do puerpério, bem como das condições do embrião/feto.

Tabela 2 - Distribuição das gestantes segundo o trimestre gestacional de início do pré-natal, conforme dados dos prontuários*

\begin{tabular}{lcc}
\hline TRIMESTRE & $\mathrm{n}$ & $\mathrm{Fr}(\mathbf{\%})$ \\
\hline Primeiro trimestre & 34 & $\mathbf{6 4}$ \\
Segundo trimestre & 10 & $\mathbf{1 9}$ \\
Terceiro trimestre & 2 & $\mathbf{4}$ \\
Não consta & 7 & $\mathbf{1 3}$ \\
\hline TOTAL & 53 & $\mathbf{1 0 0}$ \\
\hline
\end{tabular}

* Unidade Básica de Saúde no município de Ribeirão Preto, julho de 1995

Observa-se que 13\% desta amostra não apresentava registro referente ao trimestre em que a gestante iniciou o seu pré-natal. No momento da pesquisa $41 \%$ dos prontuários analisados não constavam informações sobre o trimestre gestacional em que a gestante estava naquele momento, conforme Tabela 3.

Tabela 3 - Distribuição das gestantes segundo o trimestre gestacional no momento da pesquisa, conforme dados dos prontuários*

\begin{tabular}{lcc}
\hline TRIMESTRE & $\mathrm{n}$ & Fr(\%) \\
\hline Primeiro trimestre & 10 & $\mathbf{1 9}$ \\
Segundo trimestre & 11 & $\mathbf{2 1}$ \\
Terceiro trimestre & 10 & $\mathbf{1 9}$ \\
Não consta & 22 & $\mathbf{4 1}$ \\
\hline TOTAL & 53 & $\mathbf{1 0 0}$ \\
\hline
\end{tabular}

* Unidade Básica de Saúde no município de Ribeirão Preto, julho de 1995
O registro referente ao número de gestação anteriores, em $17 \%$ dos prontuários não constavam tanto para aquelas multigestas que já freqüentavam o serviço, como para as mulheres que procuravam o serviço pela primeira vez.

De acordo com o Programa de Assistência Integral a Saúde da Mulher da Secretaria Municipal da Saúde de Ribeirão Preto ${ }^{9}$, em toda a consulta de seguimento de pré-natal deverá ser feita uma anamnese e exame físico, que constará de averiguação das condições maternas (peso corporal, pressão arterial, presença de edema etc..) e condições fetais (medida da altura uterina e circunferência abdominal, ausculta dos batimentos cardíacos e palpação fetal). A idade gestacional deverá ser avaliada em cada consulta. A atuação médica deverá abranger a orientação alimentar, prescrição de sulfato ferroso e/ou vitaminas e mineiras, tratamento de algumas patologias de menor complexidade (hiperemese, parasitose, anemia, infeção, hipertensão leve etc..) e deverá estar atento às manifestações e correção dos chamados "pequenos distúrbios da gravidez" (náusea e vômitos, pirose, alteração de hábitos intestinal, etc.), bem como, orientar quanto aos cuidados com as mamas (preparação mamilar), prevenção de varizes, estrias e aspectos relacionados à evolução da gravidez, parto e puerpério. Todos os dados obtidos deverão ser obrigatoriamente anotados na ficha obstétrica e no cartão da gestante .

Observa-se nos registros analisados um distanciamento do normativo proposto pelo Programa de Assistência Integral a Saúde da Mulher da Secretaria Municipal da Saúde de Ribeirão Preto ${ }^{9}$, da Secretaria de Estado da Saúde de São Paulo ${ }^{10,11}$ e do Ministério da Saúde $^{3}$, conforme os resultados levantados nesta pesquisa. Desta amostra $43 \%$ são primigesta, conforme tabela abaixo.

Tabela 4 - Distribuição das gestantes segundo o número de gestações, conforme dados dos prontuários*

\begin{tabular}{lcc}
\hline Número de gestação & n & Fr(\%) \\
\hline $\begin{array}{l}\text { Primigesta } \\
\text { Multige sta }\end{array}$ & 23 & $\mathbf{4 3}$ \\
Não consta & 21 & $\mathbf{4 0}$ \\
\hline TOTAL & 9 & $\mathbf{1 7}$ \\
\hline * Unidade Básica de Saúde no município de Ribeirão Preto, \\
julho de 1995 \\
\multicolumn{2}{c}{ Em 17\% dos registros não constavam informação } \\
sobre o número de gestação anterior. Para TANAKA ${ }^{12}$, \\
esta situação sugere que a supervisão do ciclo gravídico
\end{tabular}


está sendo realizada sem um adequado registro dessas condições, o que pode estar comprometendo a integralidade e a continuidade da assistência, principalmente no que diz respeito aos dados referentes à gestações anteriores.

Observa-se que dentre as multigestas, $62 \%$ tinham de um ou dois partos e $19 \%$ dos prontuários não apresentavam estes registros, conforme Tabela 5.

Tabela 5 - Distribuição das gestantes segundo o número de partos, conforme dados dos prontuários*

\begin{tabular}{lcc}
\hline Número de partos & $\mathrm{n}$ & $\mathrm{Fr}(\mathbf{\%})$ \\
\hline Uma a duas & 13 & $\mathbf{6 2}$ \\
Três a quatro & 3 & $\mathbf{1 4}$ \\
Cinco a seis & 1 & $\mathbf{5}$ \\
Não consta & 4 & $\mathbf{1 9}$
\end{tabular}

TOTAL

21

100

* Unidade Básica de Saúde no município de Ribeirão Preto, julho de 1995

Dentre estas mulheres $71 \%$ possuíam até dois filhos vivos, conforme Tabela 6 .

Tabela 6 - Distribuição das gestantes segundo o número de filhos vivos, conforme dados dos prontuários*

\begin{tabular}{lcc}
\hline Número de filhos & $\mathrm{n}$ & $\mathrm{Fr}(\mathbf{\%})$ \\
\hline Um a dois & 15 & $\mathbf{7 1}$ \\
Três a quatro & 2 & $\mathbf{1 0}$ \\
Cinco ou mais & 0 & $\mathbf{0}$ \\
Não consta & 4 & $\mathbf{1 0}$ \\
\hline TOTAL & 21 & $\mathbf{1 0 0}$ \\
\hline
\end{tabular}

* Unidade Básica de Saúde no município de Ribeirão Preto, julho de 1995

O parto anterior em $52 \%$ das gestantes foi normal, conforme Tabela 7 .

Tabela 7 - Distribuição das gestantes segundo o tipo de parto, conforme dados dos prontuários*

\begin{tabular}{lcc}
\hline Tipo de parto & $\mathrm{n}$ & $\mathbf{F r}(\mathbf{\%})$ \\
\hline Normal & 11 & $\mathbf{5 2}$ \\
Cesariana & 3 & $\mathbf{1 5}$ \\
Não consta & 7 & $\mathbf{3 3}$ \\
\hline TOTAL & 21 & $\mathbf{1 0 0}$ \\
\hline
\end{tabular}

* Unidade Básica de Saúde no município de Ribeirão Preto, julho de 1995
Quanto ao registro em relação ao tipo de parto anterior $33 \%$ dos prontuários consultados não apresentavam esta informação. Na amostra analisada 52\% tiveram parto anterior por via vaginal, salientando-se que essas pacientes utilizam os Serviços Hospitalares do Sistema Único de Saúde (SUS). Diferentemente, dos dados do Ministério da Saúde, segundo NOGUEIRA ${ }^{7}$, apontam que $44,1 \%$ dos partos realizados nos hospitais públicos e conveniados com INAMPS foram cesarianos e grande parte delas por meio de indicação abusiva.

Por ocasião da coleta de dados, $64 \%$ das gestantes tinham realizado de uma a quatro consultas de pré-natais, conforme Tabela 8 .

Tabela 8 - Distribuição das gestantes segundo o número de consultas de pré-natais, conforme dados dos prontuários*

\begin{tabular}{lcc}
\hline Número de consultas no pré-natal & n & Fr(\%) \\
\hline Uma a quatro & 34 & $\mathbf{6 4}$ \\
Cinco a nove & 13 & $\mathbf{2 5}$ \\
Dez a doze & 6 & $\mathbf{1 1}$ \\
\hline TOTAL & 53 & $\mathbf{1 0 0}$ \\
\hline
\end{tabular}

* Unidade Básica de Saúde no município de Ribeirão Preto, julho de 1995

O dados do Ministério da Saúde, segundo NOGUEIRA $^{7}$, apontam que $53 \%$ das grávidas têm um acompanhamento pré-natal de até quatro consultas durante a gestação. Assim, podemos inferir que ao término do acompanhamento de pré-natal as gestantes desta unidade poderão ter um número superior ao apresentado pelo Ministério da Saúde, uma vez que 64\% iniciaram o pré-natal no primeiro trimestre e apenas $19 \%$ estavam no terceiro trimestre no momento em que foi realizada esta pesquisa.

\section{Identificação dos procedimentos realizados na assistência Pré-Natal: Categoria II}

$\mathrm{Na}$ análise dos dados levou-se em conta: registro das condições de admissão no pré-natal, os antecedentes obstétricos/ginecológicos, dados referentes à anamnese geral e específica, exame físico geral e tocoginecológico, imunização, procedimentos diagnósticos utilizados nesse serviço (exames laboratoriais, ultra-sonografia e citológico).

Com relação aos registros referente à anamnese geral e específica, exame físico geral e tocoginecológico, as condições de admissão no pré-natal e os antecedentes obstétricos/ginecológicos, constatou-se a falta de informação completa e a presença de registro truncado e ilegível. 
Segundo as normas e recomendações do Programa de Assistência Integral a Saúde da Mulher da Secretaria Municipal da Saúde de Ribeirão Preto ${ }^{9}$, Secretaria de Estado da Saúde de São Paulo ${ }^{10,11}$ e do Ministério da Saúde $^{3}$, a confirmação da gravidez deverá ser feita através de "avaliação prévia", constando de anamnese e exame ginecológico e, se necessário, exames complementares (dosagem de gonadotrofinas na urina e ultra-sonografia).

Nos dados coletados observa-se que em $17 \%$ não constavam informações sobre solicitação de exames de ultra-sonografia, conforme Tabela 9.

Tabela 9 - Distribuição das gestantes segundo o número de exames de ultra-sonografia realizados, conforme dados dos prontuários*

\begin{tabular}{lcc}
\hline $\begin{array}{l}\text { Número de exames de ultra- } \\
\text { sonografia }\end{array}$ & n & $\operatorname{Fr}(\mathbf{\%})$ \\
\hline Não consta & 9 & $\mathbf{1 7}$ \\
Um & 40 & $\mathbf{7 5}$ \\
Dois & 4 & $\mathbf{8}$ \\
\hline TOTAL & 53 & $\mathbf{1 0 0}$ \\
\hline
\end{tabular}

* Unidade Básica de Saúde no município de Ribeirão Preto, julho de 1995

Em $75 \%$ da amostra estes exames foram solicitados apenas uma vez, porém muitas vezes sem especificar a causa desta solicitação.

De acordo com os registros, $23 \%$ dos exames de ultra-sonografia solicitados não constavam em que trimestre gestacional foram realizados, conforme Tabela 10.

Tabela 10 - Distribuição das gestantes segundo o trimestre gestacional em que foram solicitados exames de ultra-sonografia, conforme dados dos prontuários*

\begin{tabular}{lcc}
\hline Trimestre gestacional & n & Fr(\%) \\
\hline Primeiro trimestre & 27 & $\mathbf{5 1}$ \\
Segundo trimestre & 10 & $\mathbf{1 9}$ \\
Terceiro trimestre & 4 & $\mathbf{7}$ \\
Não consta & 12 & $\mathbf{2 3}$ \\
\hline TOTAL & 53 & $\mathbf{1 0 0}$ \\
\hline
\end{tabular}

* Unidade Básica de Saúde no município de Ribeirão Preto, julho de 1995
Dos exames de ultra-sonografia registrados observa-se que $51 \%$ foram solicitados no primeiro trimestre, o que provavelmente coincide com o período de diagnóstico da gestação, uma vez que, $62 \%$ destas gestantes iniciaram o pré-natal no primeiro trimestre gestacional e apenas $11 \%$ não foram solicitado ultrasonografia (primeiro trimestre).

Percebe-se hoje, uma procura maior aos serviços no início da gestação (primeiro trimestre), pois o uso da ultra-sonografia como diagnóstico gestacional sobrepõem-se ao diagnóstico clínico através do exame físico e a indicação de testes laboratoriais. Assim, as mulheres procuram mais precocemente os serviços para fins diagnósticos.

Segundo o Programa de Assistência Integral a Saúde da Mulher da Secretaria Municipal da Saúde de Ribeirão Preto ${ }^{9}$, da Secretaria de Estado da Saúde de São Paulo $^{10,11}$ e do Ministério da Saúde ${ }^{3}$, a primeira consulta de pré-natal deve ser o mais precoce possível e, nesta ocasião, deve ser feita a anamnese geral e específica, exame físico geral e tocoginecológico com coleta de citologia dupla e, quando indicado, solicitar o exame colposcópio. Observa-se, no entanto, na prática um distanciamento dos objetivos estabelecidos pelos programas citados, conforme resultados das tabelas abaixo.

Em $66 \%$ dos registros não constavam informação sobre exame citológico, conforme Tabela 11.

Segundo CARRANZA ${ }^{4}$, para aferir a qualidade da consulta pré-natal que está sendo oferecida às mulheres brasileiras, pode-se utilizar alguns indicadores, como por exemplo, taxas de mortalidade materna, índice de cesarianas, índice de tétano neonatal, porcentagem de mulheres que realizaram Papanicolau e exames das mamas, dentre outros.

Tabela 11 - Distribuição das gestantes segundo o número de exames citológicos realizados, conforme dados dos prontuários*

\begin{tabular}{lcc}
\hline Números de exames citológigos & $\mathrm{n}$ & $\mathbf{F r}(\mathbf{\%})$ \\
\hline Não consta & 35 & $\mathbf{6 6}$ \\
Um & 18 & $\mathbf{3 4}$ \\
\hline TOTAL & 53 & $\mathbf{1 0 0}$ \\
\hline
\end{tabular}

* Unidade Básica de Saúde no município de Ribeirão Preto, julho de 1995 
Dos exames citológicos registrados observa-se que $67 \%$ deles foram solicitados no primeiro trimestre, conforme Tabela 12 .

Tabela 12 - Distribuição das gestantes que colheram exames citológicos segundo o trimestre gestacional em que foram solicitados, conforme dados dos prontuários*

\begin{tabular}{lcc}
\hline Trimestre gestacional & $\mathrm{n}$ & $\mathrm{Fr}(\mathbf{\%})$ \\
\hline Primeiro trimestre & 12 & $\mathbf{6 7}$ \\
Segundo trimeste & 3 & $\mathbf{1 6}$ \\
Terceiro trimestre & 4 & $\mathbf{1 7}$ \\
\hline TOTAL & 18 & $\mathbf{1 0 0}$
\end{tabular}

* Unidade Básica de Saúde no município de Ribeirão Preto, julho de 1995

Observa-se que $62 \%$ das solicitações de exames laboratoriais foram efetuadas no primeiro trimestre gestacional, período adequado, segundo recomendação do Programa de Assistência Integral a Saúde da Mulher da Secretaria Municipal da Saúde de Ribeirão Preto ${ }^{9}$, da Secretaria de Estado da Saúde de São Paulo ${ }^{10,11}$ e do Ministério da Saúde ${ }^{3}$. Entretanto, em $23 \%$ dos prontuários não constavam o registro do trimestre gestacional em que foram solicitados os exames laboratoriais, conforme a Tabela 13.

Tabela 13 - Distribuição das gestantes segundo trimestre gestacional em que foram solicitados exames laboratoriais, conforme dados dos prontuários*

\begin{tabular}{lcc}
\hline Trimestre gestacional & $\mathrm{n}$ & $\operatorname{Fr}(\mathbf{\%})$ \\
\hline Primeiro trimestre & 33 & $\mathbf{6 2}$ \\
Segundo trimestre & 7 & $\mathbf{1 3}$ \\
Terceiro trimestre & 2 & $\mathbf{2}$ \\
Não consta & 12 & $\mathbf{2 3}$ \\
\hline TOTAL & 53 & $\mathbf{1 0 0}$
\end{tabular}

* Unidade Básica de Saúde no município de Ribeirão Preto, julho de 1995

As normas do Ministério da Saúde ${ }^{3}$, atendendo recomendação da Organização Mundial de Saúde (OMS), preconizam o comparecimento da gestante a pelo menos seis consultas pré-natais no transcorrer da gestação, bem como, a realização de exames laboratoriais de rotina, tais como: tipagem sangüínea e do fator $\mathrm{Rh}$, sorologia para sífilis (VDRL), exame de urina e dosagem de hemoglobina. Para o Programa de Assistência Integral a Saúde da Mulher da Secretaria Municipal da Saúde de Ribeirão Preto ${ }^{9}$, na primeira consulta de pré-natal deverá ser solicitado os exames de rotina : tipagem sangüínea, sedimentoscopia urinária, parasitológico de fezes, hemograma completo, VDRL (trimestral), glicemia com antecedentes (se normal repetir na $28^{\mathrm{a}}$ semana, com alteração solicitar exame de GTT) e sem antecedentes na $28^{\mathrm{a}}$ semana (se normal encerra o caso, com alteração solicitar exame de GTT).

Dentre os 207 exames solicitados aparecem em maior frequência: hematimetria e tipagem, VDRL, glicemia, urina, parasitológico de fezes.

Tabela 14 - Distribuição dos tipos de exames laboratoriais realizados pelas gestantes, conforme dados dos prontuários*

\begin{tabular}{lcc}
\hline Tipos de exames bboratoriais & $\mathbf{n}$ & $\mathbf{F r}(\mathbf{\%})$ \\
\hline Pregnosticon & 13 & $\mathbf{6}$ \\
VDRL (uma vez) & 39 & $\mathbf{1 9}$ \\
Hematimetria & 6 & $\mathbf{3}$ \\
Hematimetria + Tipagem & 39 & $\mathbf{1 9}$ \\
Toxoplasmose & 2 & $\mathbf{1}$ \\
Coombs indireto & 10 & $\mathbf{5}$ \\
Urina & 28 & $\mathbf{1 3 5}$ \\
Urina cultura & 1 & $\mathbf{0 5}$ \\
Glicemia & 44 & $\mathbf{2 1}$ \\
parasitológico & 25 & $\mathbf{1 2}$ \\
\hline TOTAL & 207 & $\mathbf{1 0 0}$ \\
\hline * Unidade Básica de Saúde no município de Ribeirão Preto, \\
julho de 1995
\end{tabular}

Observa-se segundo dados registrados nos prontuários, que 12 gestantes não fizeram nenhum exame laboratorial, embora $61 \%$ das gestantes tivessem iniciado o pré-natal no primeiro trimestre gestacional. A distribuição das 41 gestantes que realizaram exames laboratoriais, de acordo com a quantidade numérica, está apresentada na tabela 15 , verificando-se que em $73 \%$ delas foram solicitados três a cinco exames.

Tabela 15 - Distribuição das gestantes segundo o número de exames laboratoriais, conforme dados dos prontuários*

\begin{tabular}{lcc}
\hline Número de exames laboratoriais & $\mathrm{n}$ & $\mathrm{Fr}(\mathbf{\%})$ \\
\hline Um a dois & 5 & $\mathbf{1 2}$ \\
Três a cinco & 30 & $\mathbf{7 3}$ \\
Seis a mais & 6 & $\mathbf{1 5}$ \\
\hline TOTAL & 41 & $\mathbf{1 0 0}$ \\
\hline
\end{tabular}

* Unidade Básica de Saúde no município de Ribeirão Preto, julho de 1995 
Em $74 \%$ dos prontuários não constavam nenhuma informação sobre a imunização anti-tetânica, anterior ou atual. O registro da imunização anti-tetânica disponível, demonstrava que $9 \%$ das gestantes não tomaram a vacina e, se havia sido iniciada, não constava encaminhamento à sala de imunização. Em $11 \%$ dos registros havia informação que as gestantes foram vacinadas, conforme Tabela 16, mas não discriminavam a quanto tempo ou período gestacional.

Tabela 16 - Distribuição das gestantes segundo a informação referente a imunização anti-tetânica, conforme dados dos prontuários*

\begin{tabular}{lcc}
\hline Imunização anti-tetânica & $\mathrm{n}$ & $\mathbf{F r}(\mathbf{\%})$ \\
\hline Já tomou & 6 & $\mathbf{1 1}$ \\
Segunda dose & 2 & $\mathbf{4}$ \\
Reforço & 1 & $\mathbf{2}$ \\
Não tomou & 5 & $\mathbf{9}$ \\
Não consta & 39 & $\mathbf{7 4}$ \\
\hline TOTAL & 53 & $\mathbf{1 0 0}$ \\
* Unidade Básica de Saúde no município de Ribeirão Preto, \\
julho de 1995
\end{tabular}

Os registros apresentavam uma desvalorização quanto a informação da imunização anti-tetânica.

Observa-se o uso de impresso diferente no setor de imunização para o registro da aplicação desta vacina, ou seja, não faziam uso do espaço existente na carteira de gestante para esta finalidade, assim como, não efetuavam registros nos prontuários sobre a conduta adotada a esse respeito.

Segundo CARRANZA ${ }^{4}$, dados do Ministério da Saúde mostram que, “ há aproximadamente trezentos casos de tétano neonatal por ano. Este número é reflexo tanto de um controle pré-natal incompleto (cerca de $20 \%$ das mulheres grávidas estariam sendo vacinadas com toxóite tetânico ao nível nacional, uma taxa inexplicável em um país onde $61,9 \%$ das mulheres comparecem a consultas pré - natais), como de uma inadequada atenção ao parto".

Não cabe aqui uma crítica a quem fez o registro, mas à qualidade do registro que, no momento, não serve de parâmetro para qualificar o tipo de assistência que está sendo prestada e cuja memória está prejudicada.

Para TANAKA $^{12}$, aliada à despersonalização do atendimento médico, a clínica perde inconscientemente seu significado e, portanto, os registros deixam de ser feitos e o paciente passa a ser a-histórico, impessoal, sem que haja responsabilidade, ou seja, essa dilui-se no todo da estrutura dos serviços ou institucionaliza-se retirando o peso de qualquer erro.

\section{CONSIDERAÇÕES FINAIS}

Observa-se que os prontuários analisados deste serviço de atenção pré-natal apresentavam um registro em que inexiste a informação referente a anamnese geral e específica, exame físico geral e tocoginecológico e às condição clínica das gestantes, caracterizando um atendimento mais ritualístico.

Desta forma, os registros não atendiam aos objetivos propostos pelo Programa de Assistência Integral a Saúde da Mulher da Secretaria Municipal da Saúde de Ribeirão Preto ${ }^{9}$, Secretaria de Estado da Saúde de São Paulo ${ }^{10,11}$ e do Ministério da Saúde ${ }^{3}$, a saber: assegurar a boa qualidade do atendimento à saúde da mulher em suas necessidades clínicas, ginecológicas, obstétricas e mentais; identificar, prevenir e controlar os fatores de risco que possam afetar a saúde da mulher; diagnosticar e tratar o mais precocemente possível as patologias da mulher, entre outros.

$\mathrm{OBA}^{8}$ relata em seu estudo sobre assistência prénatal no município de Ribeirão Preto, que as mulheres deparam com um atendimento que consolida a assistência médica como prática hegemônica, onde as consultas são basicamente em cima de queixas e condutas, realização dos exames físicos e tocoginecológicos e solicitação de exames complementares, não havendo tempo nem espaço para fala e/ou questionamento destas mulheres. O atendimento é mais ritualístico do que preventivo ou resolutivo, embora o Programa de Assistência Integral à Saúde da Mulher ${ }^{11}$ preconize assistir às necessidades globais de saúde da mulher decorrentes de sua condição bio-psico-social, além das relacionadas ao aparelho reprodutivo, através de atividades de saúde gerais e específicas, que incluem: promoção, proteção, assistência e recuperação da saúde, de modo a assegurar a boa qualidade de atendimento à saúde da mulher quer nas suas necessidades clínicas, ginecológicas, obstétricas e mentais.

O que se observa na prática, segundo $\mathrm{OBA}^{8}$, é um distanciamento dos discursos oficiais, ou seja, a visão que se tem desta mulher está ligada aos aspectos biológicos e é fragmentada.

Segundo TANAKA ${ }^{12}$ a deficiência nos registros, refletem necessariamente uma deficiência na execução e resolução da assistência, pois pode ser que o registro não tenha sido feito por "falta de tempo" e/ou pelo não reconhecimento da importância deste registro. Se não há registro, não há memória escrita. A gestante reduz-se a um número a mais para o sistema de "cobrança". Aliado a este fato, as consultas rápidas e impessoais desse sistema fazem com que o indivíduo, ou melhor, a mulher seja anulada. O que tem mais importância é o meio pelo qual a nova tecnologia pode ser usada neste corpo para gerar conhecimento. 
THE PRECARIOUSNESS OF PRENATAL CARE RECORDS IN BASIC HEALTH UNITS IN THE CITY OF RIBEIRÃO PRETO-S.P.

This survey aimed at characterizing the population of pregnant women utilizing prenatal care services of a Basic Health Unit in the city of Ribeirão Preto, São Paulo, Brazil in July 1995 and analyzing the records of the service given to pregnant women who went to this Unit in terms of general and specific anamnesis, general and tocogynecological physical examination and the diagnostic procedures used in this service. The records showed a lack of information about patients' general and specific anamnesis, general and tocogynecological physical examination and clinical condition, characterizing a more ritualistic procedure. Therefore, records did not meet the objectives proposed by the Program for Comprehensive Women Health Care of the Ribeirão Preto City Health Department with the São Paulo State Health Department and the Health Ministry: to ensure good quality in women's care service regarding their clinical, gynecological, obstetric and mental needs; to identify, prevent, and control risk factors which may affect women's health; to diagnose and treat women's pathologies as soon as possible, among others.

KEY WORDS: care, women's health

\section{LA PRECARIEDAD DE LOS REGISTROS DE ATENCIÓN PRENATAL EN UNA UNIDAD BÁSICA DE SALUd EN EL MUNICIPIO DE RIBEIRÃO PRETO- S.P.}

El objetivo de esta investigación ha sido el de caracterizar la población de mujeres embarazadas que han ido en busca del servicio de atención prenatal en una Unidad Básica de Salud, del municipio de RIBEIRÃO PRETO, Estado de São Paulo, Brasil, durante el mes de julio de 1995. Se ha estudiado la calidad de la atención dada a las usuarias de la Unidad, en lo que respecta a la anamnesis general y especifica, el examen fisico general y tocoginecológico y los procedimentos diagnósticos utilizados en el servicio. Se observa que las historias analizadas de ese servicio de atención prenatal presentaban un registro en que se encontraban ausentes las informaciones sobre anamnesis general y especifica, examen fisico general y tocoginecológico y de la condición clínica de las pacientes, lo cual caracteriza una atención más bien ritualística. De tal manera que los registros no cumplen con los objetivos propuestos por el Programa de Atención Integral a la Salud de la Mujer, desarrollado por la Secretaría Municipal de Salud de Ribeirão Preto, Secretaría de Estado de la Salud de São Paulo y del Ministerio da Salud. Entre otros, dichos objetivos son: asegurar la calidad de la atención a la salud de la mujer en sus necesidades clínicas, ginecológicas, obstétricas o mentales; identificar, prevenir, diagnosticar y controlar los factores de riesgo que pudieran afectar la salud de la mujer; y diagnosticar y tratar lo más tempranamente posible las patologías de la mujer.

TÉRMINOS CLAVES: asistencia, salud de la mujer

\section{REFERÊNCIA BIBLIOGRÁFICA}

01. BARDIN, L. Análise de conteúdo. Lisboa, Edições 70, 1977.

02. BRASIL. Ministério da Saúde. Secretaria de Assistência à Saúde. Departamentos de Programas de Saúde. Coordenação de Saúde Materno Infantil. Manual dos Comitês de Mortalidade Materna. Brasília, 1994.

03. Ministério da Saúde. Assistência Integral à Saúde da Mulher: base de ação programática. Brasília: Centro de Documentação do Ministério da Saúde, 1984.

04. CARRANZA, M. Saúde reprodutiva da mulher brasileira. In: Mulher brasileira é assim. Rio de Janeiro: Rosa dos Tempos, 1994. p. 108-109

05. DUGAS, B.W. Enfermagem prática. Rio de Janeiro: Interamericana, 1984.
06. LAURENTI, R. Uma questão de saúde e discriminação. Jornal da USP- Opinião: n. 2, p. 23 - 29, outubro 1995.

07. NOGUEIRA, M.I. Assistência pré-natal: prática de saúde a serviço da vida. Rio de Janeiro: Hucitec, 1994.

08. OBA, M. D. V. Assistência pré-natal: uma assistência integral ou fragmentada? Ribeirão Preto, 1996. Dissertação (Mestrado) - Escola de Enfermagem de Ribeirão Preto, Universidade de São Paulo.

09. RIBEIRÃO PRETO. Secretaria Municipal de Saúde de Ribeirão Preto. Programa de assistência Integral à Saúde da Mulher. Ribeirão Preto, 1993.

10. SÃO PAULO. Secretaria de Estado da Saúde de São Paulo. Subprograma de Saúde da Mulher. Subsídios e Diretrizes. São Paulo: Grupo de Saúde da Mulher, 1986. 
11. Secretaria de Estado da Saúde de São Paulo. Subprograma de Saúde da Mulher. Pré-Natal Normal, volume II. São Paulo: Grupo de Saúde da Mulher, 1986.
12. TANAKA, A.C.d'A. Maternidade: dilema entre nascimento e morte. São Paulo: Hucitec, 1995. 13. TESTA, M. et al. Pensar em saúde. Porto Alegre: Artes Médicas, 1992. 